\title{
THE PHYSICAL BASIS OF TRANSPARENCY OF THE NORMAL CORNEA
}

\author{
TIMOTHY J. FREEGARD \\ Plymouth
}

\section{SUMMARY}

An understanding of the physical basis of corneal transparency has been a subject of interest amongst physicists, basic scientists and ophthalmologists. Impairment of corneal clarity is a significant cause of visual morbidity worldwide. Several highly mathematical treatises have been presented in support of different theories of corneal transparency in the normal cornea relating structure to function, particularly in the years since the advent of the electron microscope, which has brought about a much greater understanding of corneal ultrastructure. This review presents the concepts that underpin the theories of corneal transparency.

The cornea forms part of the structural coat of the eye. As well as being tough, it must be transparent and maintain a smooth and stable curvature since it contributes to the major part of the focusing power of the eye. Whilst those properties can be readily appreciated clinically, contention has arisen as to how transparency is achieved in the light of increasing knowledge of the cornea's structure and other physical properties. Further, transparency is affected by changes in hydration, although this is controlled within fine limits in the normal state. ${ }^{1,2}$

In approaching the question as to what characteristics enable the cornea to be transparent, it is necessary to consider both corneal structure and the possible ways in which electromagnetic radiation may behave on confronting different media.

\section{CORNEAL STRUCTURE}

The cornea occupies the anterior one-fourteenth of the fibrous tunic of the eye and approximates a diverging meniscus lens in shape, its internal concave surface being steeper than its external convex surface. It comprises a stroma, constituting $90 \%$ of its thickness, bounded by anterior and posterior

Correspondence to: Timothy J. Freegard, FRCS, FRCOphth, MD, Royal Eye Infirmary, Apsley Road, Plymouth, Devon PL4 6PL, UK. Tel: +44 (0)1752 203142. limiting membranes with their associated cellular layers, the epithelium and endothelium respectively. The central thickness of the human cornea is approximately $0.5 \mathrm{~mm}$, being $0.69 \mathrm{~mm}$ at the periphery.

The stroma comprises over 200 sheets of lamellae, each 1-2 $\mu \mathrm{m}$ thick, of collagen fibrils approximately $36 \mathrm{~nm}$ in diameter. ${ }^{3,4}$ The arrangement of fibrils within a given lamella is highly ordered such that they lie in parallel rows. Keratocytes, or corneal fibroblasts, are interspersed between the lamellae forming an interlinking network throughout the cornea, occupying between $3 \%$ and $5 \%$ of the stromal volume. Bowman's layer, the anterior portion of the stroma that is between 8 and $12 \mu \mathrm{m}$ thick, is morphologically distinct and comprises a closepacked and randomly orientated arrangement of collagen fibres devoid of keratocytes. Individual fibres are generally smaller than those in the remainder of the stroma. ${ }^{5-7}$

The corneal stroma has a powerful tendency to imbibe water and swell because of the hydrophilic nature of the proteoglycan component of the matrix surrounding the collagen fibrils. ${ }^{8,9}$ The spatial arrangement of collagen fibrils in the cornea is believed to be dependent upon their dense coating of acid glycosaminoglycan sub-units, predominantly keratan sulphate, which maintains their separation. The glycosaminoglycans create a negatively charged field surrounding each collagen fibril. In the peripheral cornea, the larger chondroitin sulphate molecule is believed to be responsible for the wider spacing of collagen fibrils found in this region. The sclera contains different proteoglycans again, with dermatan sulphate and hyaluronic acid predominating, which contribute to its very different arrangement of fibrils. ${ }^{10}$

In addition, the cell covered surfaces, in particular endothelial, are intimately linked to transparency since they are responsible for maintaining the state of relative deturgescence of the stroma through active 
transport. ${ }^{1,11,12}$ Swelling of the epithelium itself from imbibition of fluid following mechanical or metabolic trauma has a profound effect on transparency. ${ }^{13}$

\section{BEHAVIOUR OF ELECTROMAGNETIC RADIATION}

When electromagnetic radiation, including light, confronts a different medium it may be modified in several ways. ${ }^{14}$ Light may be:

1. Unaffected - continuing undeviated along the same axis as the incident beam. This only strictly occurs along the optical axis at a regular surface separating transparent media.

2. Refracted - continuing in a forward direction but at an angle to the original axis. This implies that the beam is passing between transparent media of different refractive indices at a regular (smooth) interface at an angle other than the normal.

3. Reflected - either according to the laws of reflection at a plane surface or diffusely.

4. Absorbed - where the incident energy is converted into heat or fluorescence where light of a longer wavelength is emitted relative to that absorbed.

5. Scattered - all the light passing to the medium emerges but with random direction.

At least three types of scatter occur:

i. Random reflection, where particles suspended in the medium act as tiny mirrors causing random reflection as a result of their random orientation with respect to the incident beam. This occurs when the particles are large relative compared with the wavelength of light and light is predominantly back-scattered (back towards the source).

ii. Diffraction, where the particles suspended in the medium are small relative to the wavelength of light. Such particles act as centres of radiation, scattering light in all directions.

iii. Diffuse refraction, where fluctuations in refractive index occur throughout the medium such that the direction of light travel is deviated but in a predominantly forward direction.

These effects are inevitably compounded since many interactions can occur during light passage through a medium containing many scattering particles. The only truly non-scattering medium is a vacuum not influenced by gravity.

Further efforts may occur attributable to electromagnetic wave theory.

In the special circumstance where light strikes a regular grating in which the separation of its constituent slits is of the same order of magnitude as the wavelength of incident light, the light may be dispersed in a regular manner to produce a diffraction pattern or spectrum. In addition, the light can be selectively transmitted or reflected so that the orientations of individual light waves are no longer random but 'polarised'.

All these phenomena take place to some extent when light strikes the eye. The properties of simple reflection and refraction are well understood in terms of geometrical optics and are relatively simple concepts since they occur at any regular surface separating transparent media of differing refractive indices. On the question of transparency, which involves interaction of light with the internal structural components of a given medium, it is relatively unimportant that the transmitted intensity is reduced to a small proportion of the incident intensity by reflection or absorption. However, it is essential that only a very small proportion of the light should be scattered in the forward direction relative to that transmitted if a clear image is to be formed. These points can be readily appreciated by looking through a neutral density filter - a clear though darkened image is obtained since light is absorbed uniformly across the visible spectrum.

\section{THEORIES OF TRANSPARENCY}

The transparency of the ocular media of the eye was originally ascribed to the homogeneous nature of their constituent elements ${ }^{15}$ and the absence of opaque structures, especially blood vessels. ${ }^{16}$ Although it has long been recognised that the cornea comprises fibrous units and an interstitial fluid, its transparency was accounted for by all its components having identical refractive indices and by its relative acellularity. ${ }^{17}$ Although this view was widely accepted, other observers suggested that the refractive indices of the various elements could be different, but postulated that surface refraction between them was reduced by the close apposition of fibrils, ${ }^{18}$ or by the small amount of fluid component separating them. ${ }^{19}$

Measurement of refractive index has been attempted in isolated components of the cornea. Aurell and Holmgren ${ }^{20}$ found values of 1.547 for dry collagen and 1.342 for pressure-extracted corneal fluid derived from the interstitium. Some investigators believe that such separation is unlikely to be representative of the tissues in vivo, giving values too low for the ground substance and too high for the collagen fibrils. ${ }^{21}$ However, the observed property of birefringence (i.e. having different refractive properties in different directions) supports the view that differences in refractive index exist throughout the cornea. $^{22-28}$ It should be pointed out that the presence of such background or inherent birefringence (as opposed to stress birefringence induced by abnormal mechanical forces) has only been observed in isolated corneal preparations and may not be representative of normal corneal stroma in vivo. The 
question has arisen as to just how transparency is attained in a heterogeneous medium of fibres embedded in a matrix of different refractive index.

Caspersson and Engström ${ }^{29}$ suggested that transparency was a result of the alignment of rows of fibrils within the stroma, with the diameter of the fibrils corresponding to a peak in the absorption spectrum of the cornea at $300 \mathrm{~nm}$. Light rays were believed to pass down rows of fibrils, being refracted in transit, to finally emerge undeviated. Reflection at the fibril surfaces was thought to be limited by gradation in refractive index between the collagen fibrils and ground substance which they noted from their own measurements. This theory was considered unsatisfactory since it did not take account of light rays entering the cornea other than perpendicularly and the alleged fibril size was an order of magnitude different from the true value of $36 \mathrm{~nm}$.

Following electron microscopic studies, Schwarz ${ }^{30}$ and Davson ${ }^{31}$ reasserted the theory of uniform refractive index believing that the mucopolysaccharide substance occupying the spaces between fibrils raises the refractive index of the interstitium to that of collagen.

Maurice $^{26}$ looked in depth at the question of corneal transparency. He assumed that there was a sharp difference in refractive index between collagen fibrils and ground substance, based on measurements of birefringence, and indicated that if individual collagen fibrils scattered light independently of one another the stroma would be opaque. His theory asserted that, since the cornea is transparent, a phase relationship must exist between electric fields emanating from individual fibres, resulting in destructive interference of scattered wavelets which limited the intensity of light scattering. He believed that this distribution of fibrils was the only arrangement that

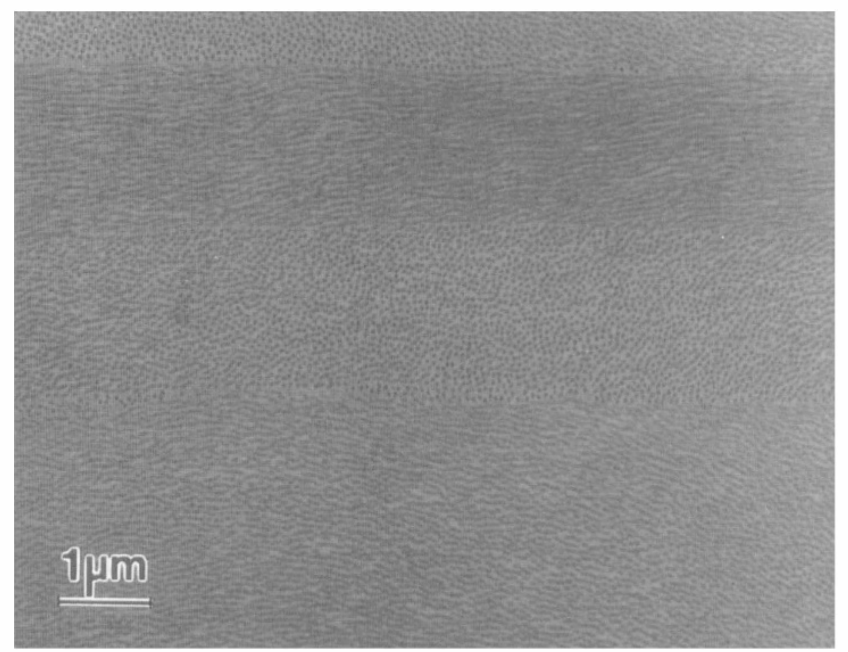

Fig. 1. Section through two adjacent lamellae of human cornea. The ordered arrangement of individual collagen fibrils can be seen in both transverse and longitudinal section. could ensure transparency on a simple theoretical basis and was in accordance with the striking electron micrographic appearances (Fig. 1). The theory initially assumed a high degree of uniformity in size of corneal fibrils and a high degree of regularity in their arrangement as a lattice. Some investigators found fibril diameters to vary threefold within the same cornea ${ }^{32}$ and that the fibrils did not appear to be disposed in a perfect lattice..$^{30,33}$ The discrepancy was originally attributed to processing artefact in samples prepared for electron microscopy.

This theory was widely accepted since it was supported by other experimental findings. The measured refractive index of extracted ground substance was close to the value obtained from Maurice's calculations. The observation that decreased transparency followed increased hydration could be explained by a disordering of the lattice arrangement. Rapid loss of transparency associated with point pressure could be explained by local disordering of the lattice, disappearing immediately on removal of the distorting force.

Maurice constructed a sonic analogue in support of his theory. ${ }^{26} \mathrm{He}$ demonstrated that sound of a specific frequency (therefore wavelength in air) when passed through a lattice of steel rods was diminished in amplitude following disordering of the regular lattice arrangement.

However, there are some shortcomings to this lattice theory. Several investigators considered electron micrographs to be representative of the tissue to the extent that the explanation of corneal transparency could not derive from a perfect lattice arrangement of fibrils. ${ }^{21.32-35}$ The regularity of spacing believed to be necessary for the theory is not present in other transparent ocular structures. ${ }^{36}$

Smith $^{21}$ indicated that supposed measurements of refractive index of ground substance were likely to be inappropriately low in value since important components affecting refractive index failed to be extracted from the cornea by the pressing technique used by Aurell and Holmgren. ${ }^{20,37} \mathrm{He}$ considered the collagen fibrils to be more hydrated and hence to have a lower refractive index than that found in dry collagen. He believed that the factors making the cornea highly transparent were the very small size of the collagen fibrils and the close similarity between refractive indices of fibrils and ground substance.

Goldman and Benedek, ${ }^{34}$ whilst studying the morphological basis for the inability of the dogfish cornea to swell, noted that the central Bowman's zone occupied about $15 \%$ of the total corneal thickness. The fibres of Bowman's zone in the dogfish are neither parallel nor disposed in a lattice and yet it scatters less light than the rest of the corneal stroma. In this instance, the fibres could not be regarded as independent point-scatterers. Gold- 
man and Benedek concluded that a lattice arrangement of collagen fibrils was not a necessary condition for corneal transparency, and that transparency existed in Bowman's zone since it is optically homogeneous over distances comparable to approximately half the wavelength of light. They also observed that scleral collagen fibrils were of very variable diameters $(25-480 \mathrm{~nm})$ and that the separation of adjacent fibre bundles was such that the intervening spaces were large relative to the wavelength of light, and suggested that these features were more important than the lack of a lattice arrangement in accounting for scleral opacity.

In a subsequent paper, ${ }^{38}$ these same authors supported this theory further by alluding to diffraction theory expressed in terms of geometrical optics. According to the theory of diffraction, periodic fluctuations in refractive index over distances small compared with half the wavelength of light do not produce scattering. Expressed in simple terms, light cannot resolve structures substantially smaller than the dimension of its wavelength. They showed that in swollen corneas with impaired transparency there were 'lakes' devoid of fibrils generally greater than half the wavelength of light and postulated that such areas were likely to produce corresponding fluctuations in the refractive index. They also suggested that Maurice's description essentially produces a medium whose index of refraction is homogeneous over dimensions comparable to the wavelength of light. The term 'homogeneity' only has meaning if the scale of the medium being studied is defined: if considered on a small enough scale, no medium is homogeneous since it is made up of different molecules and atoms. Benedek $^{39}$ summarised the theory by stating that scattering of light is only produced by those fluctuations in refractive index whose Fourier components ${ }^{40}$ vary over distances equal to or larger than half the wavelength of light in the medium.

Experiments performed by Cox et $a l^{32}$ have indicated that fibril diameters could vary considerably both theoretically and experimentally and that a regular crystalline structure is not necessary to enable transparency provided that the fibril diameters remain a small proportion of the wavelength of light. They demonstrated that loss of transparency following corneal swelling was associated with a decrease in the order of collagen fibril spacing.

Feuk $^{41}$ considered the theoretical relationship between wavelength and different light-scattering mechanisms. Since this relationship changes according to the mechanism of scattering, he suggested that such an experimental investigation would provide useful information in understanding the optical properties, particularly of the normal cornea. He suggested that the requirement for such strict regularity of fibrils in Maurice's lattice theory was not necessary and that a displacement from ideal fibril positions of up to one-third of a fibril diameter would produce only $1 \%$ scatter. In a subsequent study by Farrell et al. ${ }^{42}$ it was found that measurements of light scattering supported the theory of a short-range ordering of collagen fibrils rather than a strict lattice arrangement and that the scattering caused by swollen corneas was consistent with large optical inhomogeneities in regions completely devoid of fibrils as seen by electron microscopy.

None of the theories of corneal transparency adequately explains the reversible corneal stromal opacification that can be observed during a local pressure load or marked increase in intraocular pressure. This phenomenon is much more apparent when the stroma is overhydrated. It may merely represent exaggerated stress lines (i.e. an extreme form of stress birefringence) similar to that seen in otherwise transparent synthetic plastic materials when distorted.

Several investigators have studied diffraction patterns of corneas in the belief that this provides an insight into normal corneal structure..$^{24,25,28,43}$ This view follows from the observation that the phase correlation of scattered light and X-rays from a regular grating can be related to the spacings of the grating. Initial interpretations led to some contradictory and confusing results, at least partly because tissue needed to be isolated for considerable periods of time, particularly with standard X-ray diffraction, and was probably not representative of tissue in its normal physiological state. Bettelheim ${ }^{22,23}$ studied small-angle light-scattering patterns occurring in corneas of humans and other species. Differences are apparent in such patterns but the structural implications of this are unknown since electron microscopic ultrastructural appearances may often be very similar. Kikkawa ${ }^{24,25}$ believed the rabbit cornea to behave as a simple grating with $14 \mu \mathrm{m}$ spacing, which he postulated to be due to a superstructure of 'fibres' composed of the ordered collagen fibrils and hence proposed a 'double lattice' theory to describe these two apparent levels of organisation. This was not substantiated by histological evidence, and the observation was thought to be due to an artefactual waviness of lamellae of isolated corneas in the absence of intraocular pressure. ${ }^{44}$ McCally and Farrell ${ }^{27,45}$ sought to limit the effect of these undulating lamellae by applying a normal pressure gradient across the experimental cornea. They found that the orientation of lamellae across the rabbit cornea is not random but follows preferentially one of two orthogonal directions. There is support for this finding from a synchrotron $\mathrm{X}$-ray diffraction study, ${ }^{46}$ but more recent electron microscopic study of human corneas suggests no such 
arrangement, with successive lamellae crossing at random angles to one another. ${ }^{47}$

Vaezy and Clark ${ }^{48}$ performed a quantified analysis of Fourier components of the density fluctuations in the sclera and cornea with digitised electron microscopic images of cornea and sclera. Results show that the Fourier components responsible for opacity of the sclera have sizes of the order of the wavelength of light $(300 \mathrm{~nm})$, whereas those in transparent corneas are much smaller $(80 \mathrm{~nm})$. The major structural difference between these tissues is the size and spacing of the constituent collagen fibrils.

\section{MEASUREMENT OF CORNEAL LIGHT TRANSMISSION, ABSORPTION AND SCATTER}

The normal cornea is highly transparent to light in the visible range. Actual measurements of transmission require placement of a light source one side of the cornea and a light detector on the other side, and accordingly all such studies for cornea alone have been performed in vitro. A further difficulty encountered is that such measurement fails to distinguish scattered light from that absorbed. Recent studies ${ }^{49,50}$ have suggested that a high proportion of incident light (over $80 \%$ at all visible wavelengths) is transmitted by the cornea, but it should be noted that the light-detecting photodiode used for these measurements was placed very close to the cornea, thus minimising effects of scatter. Spectrophotometric measurement indicates that corneal transmission diminishes rapidly below $400 \mathrm{~nm}$ wavelength and ceases below $300 \mathrm{~nm}$ (Fig. 2). It can be deduced that much of this diminution is a result of increased short-wavelength scattering, but the complete absence of transmission below $300 \mathrm{~nm}$ can be predicted from scrutiny of the cornea's chemical components, which act as chromo-

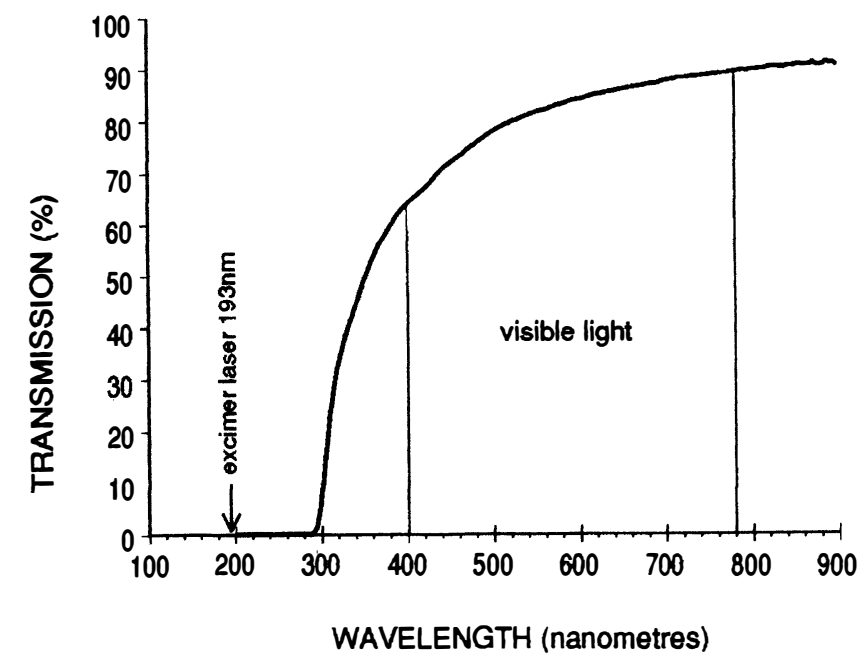

Fig. 2. Light transmission spectrum across a normal 61year-old human cornea as measured by spectrophotometry (author's own data). phores (those molecular moieties responsible for absorbing specific wavelengths of electromagnetic radiation). Ninety-nine per cent of the chemical composition of the cornea consists of molecules that contain chromophores absorbing ultraviolet light in the region between 200 and $295 \mathrm{~nm}$. Water and carbohydrate will absorb ultraviolet wavelengths below 230-235 $\mathrm{nm}$. The nucleic acids absorb between 250 and $280 \mathrm{~nm}$. However, proteins absorb ultraviolet light in two spectral bands: between 235 and 250 $\mathrm{nm}$ for aliphatic amino acid moieties and between 270 and $295 \mathrm{~nm}$ for their aromatic counterparts. ${ }^{51}$ Lerman $^{52}$ found a decreasing amount of light transmission in human corneas of increasing age in vitro which he accounted for by the generation of chromophores in the cornea. A light transmission study by Beems and van Best ${ }^{49}$ failed to demonstrate any age dependency of corneal transmission.

In practical terms, it seems unlikely that absorption plays any significant role in attenuating light transmission by the cornea in the visible range since chromophores act by absorbing specific wavelengths to produce specific absorption bands, and if significant absorption were to occur, the cornea would appear discoloured according to the balance between wavelengths absorbed and transmitted.

Several investigators have studied back-scattered light from the cornea using different techniques though without redress to specific wavelengths. Lindström $^{53}$ found that the greatest intensity of scattered light came from those regions closest to the limiting layers in rabbit corneas, in particular anteriorly, believing this to be a result of the less regular arrangement of collagen fibrils. Olsen ${ }^{54}$ measured the angular scattered light in vivo across the full thickness of central human corneas and found an increase with age presumed to be due to disordering of collagen fibrils. Smith et al. ${ }^{55}$ applied the principle of Scheimpflug photography to the assessment of corneal light scatter. With this technique, a graphical representation is made of the grey levels occurring across a photograph of an optical section produced by a slit beam of light shone obliquely across the media of the eye. Troughs correspond to dark areas and peaks to light areas, the latter indicative of amounts of scatter. One of the main difficulties with the technique is ensuring that optical sections as nearly identical as possible are obtained to enable valid comparison within the same and between different corneas. Their results accorded with those of Lindström but it should be noted that although the greatest intensities of scattered light originate close to the limiting membranes, most corneal light scatter is produced by the stroma itself. $^{45}$ 


\section{CONCLUSION}

The transparency of the normal cornea was originally ascribed to equal refractive indices of the structural components, but this theory was subsequently questioned because of apparent differing refractive indices and improved knowledge of the fine structure. The striking electron micrographic appearances of the fibril arrangement led many investigators to support a lattice theory of transparency, but the lack of such a structure in other transparent tissues and closer scrutiny of normal and abnormal corneas has prompted a reappraisal. Current understanding of corneal transparency favours a short-range ordering of fibril structure such that fluctuations in refractive index fall well within the minimum dimensions of the wavelength of light thus enabling a high degree of transparency to the visible portion of electromagnetic radiation. The highly aligned fibril structure probably has more to do with tectonic function by allowing the greatest number of fibrils per unit volume for optimal strength and in helping to maintain regularity of the surface curvature of the cornea.

Key words: Cornea, Transparency, Light transmission, Light scatter.

\section{REFERENCES}

1. Candia OA. Fluid and electrolyte transport in corneal transparency. Mt Sinai J Med 1980;47:74-9.

2. Duane TD. The steady state of corneal hydration. Am J Ophthalmol 1942;32:203-7.

3. Craig AS, Robertson JG, Parry DAD. Preservation of corneal collagen structure using low-temperature procedures for electron microscopy. J Ultrastruct Mol Struct Res 1987;96:172-5.

4. Sayers Z, Koch MHJ, Whitburn SB, Meek KM, Elliott GF, Harmsen A. Synchrotron X-ray diffraction study of the corneal stroma. J Mol Biol 1982;160:593-607.

5. Gray's anatomy. 37th ed. London: Churchill Livingstone, 1989:1181-5.

6. Klyce SD, Beuerman RW. Structure and function of the cornea. In: Kaufman HE, Barron BA, McDonald MB, Waltman SR, editors. The cornea. 1987: 1-47.

7. Jakus MA. The fine structure of the human cornea. In: Smelser GK, editor. VII International Congress of Anatomists. New York: Academic Press, 1961:343-66.

8. Harris JE. Factors influencing corneal hydration. Invest Ophthalmol Vis Sci 1962;1:151-7.

9. Hart WM. Hydration and transparency of the cornea. Am J Ophthalmol 1947;30:1022-4.

10. Borcherding MS, Blacik LJ, Sittig RA, Bizzell JW, Breen M, Weinstein HG. Proteoglycans and collagen fibre organisation in human corneoscleral tissue. Exp Eye Res 1975;21:57-70.

11. Davson H. The cornea. In: Physiology of the eye. 5th ed. London: Macmillan Press, 1990:105-38.

12. Davson $\mathbf{H}$. The hydration of the cornea. Biochem $\mathbf{J}$ 1955;59:24-8.

13. Potts AM, Friedman BC. Studies on corneal transparency. Am J Ophthalmol 1959;48:480-7.

14. Boettner EA, Wolter JR. Transmission of the ocular media. Invest Ophthalmol Vis Sci 1962;1:776-83.
15. Duke-Elder S. Textbook of ophthalmology, vol 1. Biological optics; the absorption of radiant energy by the ocular tissues. London: Kimpton:1932:809-18.

16. Fuchs's textbook of ophthalmology. 8th ed. USA: Duane Lippincott, 1919:72-3.

17. Leber $\mathrm{T}$. The circulation and nutritional relations to the eye. In: Graefe-Saemisch Handbuch der Gesampten Augenheilkunde. Vol. 2. Leipzig: Engelmann, 1903:101-491.

18. Bowman W. In: Lectures on the parts concerned in the operations on the eye and on the structure of the retina. London: Longman, Brown, Green and Longman's, 1849:1-143.

19. Kinsey VE, Cogan DG. The cornea: physiological aspects. Arch Ophthalmol 1942;28:661-9.

20. Aurell G, Holmgren H. On the metachromatic staining of corneal tissue and some observations on its transparency. Acta Ophthalmol (Copenh) 1953;31: $1-27$.

21. Smith JW. The transparency of the corneal stroma. Vision Res 1969;9:393-6.

22. Bettelheim FA, Kumbar M. An interpretation of small angle light scattering patterns of human cornea. Invest Ophthalmol Vis Sci 1977;16:233-6.

23. Bettelheim FA, Magrill R. Small angle light scattering patterns of corneas of different species. Invest Ophthalmol Vis Sci 1977;16:236-40.

24. Kikkawa Y. Diffraction spectra produced by rabbit cornea. Jpn J Physiol 1958;8:138-47.

25. Kikkawa Y. Light scattering studies of the rabbit cornea. Jpn J Physiol 1960;10:292-302.

26. Maurice DM. The structure and transparency of the cornea. J Physiol (Lond) 1957;136:263-86.

27. McCally RL, Farrell AR. Structural implications of small angle light scattering from cornea. Exp Eye Res 1982;34:99-113.

28. Stanworth A, Naylor EJ. Polarised light studies of the cornea. I. The isolated cornea. J Exp Biol 1953;30:160-3.

29. Caspersson T, Engström A. The transparency of corneal tissue. Nord Med 1946;30:1279-82.

30. Schwarz W. Electronenmikroskopische Untersuchungen über den Aufbau der Sklera und der Cornea des Menschen. Z Zellforsch 1953;38:26-49.

31. Davson H. Anatomy and physiology of the cornea. In: Rycroft BW, editor. Corneal grafts. London: Butterworth, 1955:31-5.

32. Cox JL, Farrell RA, Hart RW, Langham ME. The transparency of the mammalian cornea. J Physiol (Lond) 1970;210:601-16.

33. Schwarz W, Graf Keyserling K. Über die Feinstruktur der menschlichen Cornea, mit besonderer Berücksichtigung des Problems der Transparenz. Z Zellforsch 1966;73:540-8.

34. Goldman JN, Benedek GB. The relationship between morphology and transparency in the non-swelling corneal stroma of the shark. Invest Ophthalmol Vis Sci 1967;6:574-600.

35. Hart RW, Farrell RA. Light scattering in the cornea. J Opt Soc Am 1969;59:766-76.

36. Potts AM. Some aspects of the interrelation between corneal hydration and transparency. Invest Ophthalmol Vis Sci 1962;1:163-9.

37. Aurell G, Holmgren H. Metachromasia of the cornea, with special reference to the question of transparency. Nord Med 1946;30:1277-9. 
38. Goldman JN, Benedek GB, Dohlman CH, Kravitt B. Structural alterations affecting transparency in swollen human corneas. Invest Ophthalmol Vis Sci 1968;7: 501-19.

39. Benedek GB. Theory of transparency of the eye. Appl Opt 1971;10:459-73.

40. Champney DC. Fourier transforms and their physical applications. London: Academic Press, 1973.

41. Feuk T. On the transparency of the stroma in the mammalian cornea. IEEE Trans Biomed Eng 1970;17:186-90.

42. Farrell RA, McCally RL, Tatham PE. Wavelength dependencies of light scattering in normal and cold swollen rabbit corneas and their structural implications. J Physiol (Lond) 1973;233:589-612.

43. Naylor EJ. Polarised light studies of corneal structure. Br J Ophthalmol 1953;37:77-84.

44. Gallagher B, Maurice D. Striations of light scattering in the corneal stroma. J Ultrastruct Mol Struct Res 1977;61:100-14.

45. McCally RL, Farrell AR. The depth dependence of light scattering from the normal rabbit cornea. Exp Eye Res 1976;23:69-81.

46. Meek KM, Blamires T, Elliott GF, Gyi TJ, Nave C. The organisation of collagen fibrils in the human corneal stroma: a synchroton X-ray diffraction study. Current Eye Res 1987;6:841-6.
47. Komai Y, Ushiki T. The three dimensional organisation of collagen fibrils in the human cornea and sclera. Invest Ophthalmol Vis Sci 1991;32:2244-58.

48. Vaezy S, Clark JI. A quantitative analysis of transparency in the human sclera and cornea using Fourier methods. J Microsc 1990;163:85-94.

49. Beems EM, van Best JA. Light transmission of the cornea in whole human eyes. Exp Eye Res 1990; 50:393-5.

50. van Best JA, Bollemeijer JG, Sterk CC. Corneal transmission in whole human eyes. Exp Eye Res 1988;46:765-8.

51. Lerman S. Radiant energy and the eye. New York: Macmillan, 1980:43-59.

52. Lerman S. Biophysical aspects of corneal and lenticular transparency. Curr Eye Res 1984;3:3-14.

53. Lindström JI, Feuk T, Tengroth B. The distribution of light scattered from the rabbit's cornea. Acta Ophthalmol (Copenh) 1973;51:656-69.

54. Olsen T. Light scattering from the human cornea. Invest Ophthalmol Vis Sci 1982;23:81-6.

55. Smith GT, Brown NA, Shun-Shin A. Light scatter from the central human cornea. Eye 1990;4:584-8. 